\title{
Boundary element solution of thermal creeping flow in a nano single mixer
}

\author{
C. Nieto ${ }^{1}$, H. Power $^{2} \&$ M. Giraldo ${ }^{1}$ \\ ${ }^{1}$ Universidad Pontificia Bolivariana, Grupo de Energía y \\ Termodinámica, Facultad de Ingeniería Mecánica, Colombia \\ ${ }^{2}$ Faculty of Engineering, Department of Mechanical, Materials and \\ Manufacturing Engineering, The University of Nottingham, UK
}

\begin{abstract}
In order to employ continuum models in the analysis of the flow behaviour of a viscous Newtonian fluid in micro scale devices, it is necessary to consider at the wall surfaces appropriate slip boundary conditions instead of the classical non-slip condition. The slip behaviour in the case of micro fluid flow of rarefied gases is associated with the combined effect of reduction in momentum transfer due to the reduction in the number of molecules (shear creep) and the thermal creep or transpiration, which as a consequence of inequalities in temperatures, forces the fluid to slide over a surface from colder to hotter regions. In this work a boundary integral equation formulation for Stokes slip flow, based on the normal and tangential projection of the Green's integral representational formulae for the Stokes velocity field, which directly incorporates into the integral equations the local tangential shear rate and heat flux at the wall surfaces, is presented. The tangential heat flux is evaluated in terms of the tangential gradient of the temperature integral representational formulae presenting singularity of the Cauchy type, which are removed by the use of an auxiliary field. These formulations are used to simulate a Single rotor mixer and analyze the combined effect of both shear and thermal creep effects over mixer performance.
\end{abstract}

Keywords: linear slip boundary conditions, boundary element method, rotating mixers, thermal creep. 


\section{Introduction}

Mixing of reactants in integrated micro fluidic systems is an important issue, due to the role played in chemical or biological analysis. A variety of micro mixers has been recently developed; Mansur et al. [1] and Hessel et al. [2] present a complete review of the use and development of active and passive micro mixers. The common conclusion of both reviews relies in the lower mixing rate present in micro mixers, because of the dominant viscous laminar flow conducing to slowly mixing rates. Different mechanisms and configurations have been implemented to achieve better mixing conditions, but the majority employ active mixers regardless of the simple fabrication technology and the easy implementation of passive mixers. Examples of active mixers include the swirl micro mixer [3], oscillating flat plate stirrer [4] and vortex pump mixer [5], while for passive mixers it is possible to find simpler versions, such as the $\mathrm{Y}$ and $\mathrm{T}$ type flow [6] and more complex ones, such as chaotic micro mixers [7]. Active mixers could employ different stirrer shapes (concentric or eccentric symmetric and asymmetric rotors) to generate secondary flow, swirling flow and vortices, which enhance mixing performance [1].

When geometry devices are scaled down, the surface-to-volume ratio increases dramatically so that the surface related phenomena become increasingly dominant, e.g. micro heat exchangers and micro mixers present higher heat and mass transfer rates than macro systems of equal capacity [3]. Some new features emerge when mechanical structures are sufficiently small, and it becomes important to understand the various types of interactions that arise between the fluid flow constituents and the solid surfaces that contain them. For instance, a phenomenon known as the slip flow regime could emerges as the consequence of an insufficient number of molecules in the sampling region [8] or hydrophilic and hydrophobic recovering quality of surfaces in contact with fluids [9]. Neto et al. [10], in a review article, highlight the need of properly describing the flow near the fluid-solid interfaces, because of its relevance to a wide range of applications, from lubrication to micro fluidics.

In the case of micro fluid flow of rarefied gases, the thermal creep or transpiration also appears as a consequence of inequalities in temperatures, which forces the fluid to slide over a surface from colder to hotter regions [11]. Rarefied gas flows are generally associated with low-density conditions, such as high-altitude and vacuum. However, the small length scales commonly encountered in micro fluid flows imply that rarefaction effects will be important at much higher pressures, for more details see [12].

Taking into account the advantages of the BIMs for the numerical simulation of micro fluid flow under the slip condition, this work presents a numerical approach based on the use of the normal and tangential projections of the velocity integral representation formula for Stokes flows, resulting in weakly singular mixed system of integral equations of the first and second kinds for the normal and tangential components of the surface traction. The proposed approach is used to study flows in a Single rotor mixer (see Figure 1) under linear slip conditions with the thermal jump effect at the surface. The 
development of these formulations permits the inclusion of linear slip conditions into the boundary integral expressions directly, allowing the evaluation of micro flow in plane and curved geometries not subjected to symmetry conditions. The type of rotor mixer analyzed is of the single-shaft mixer type with an impeller rotating in close proximity to a stationary housing.

\section{Governing equations}

Fluid flow in micro scale devices usually happens at a very low Reynolds number due to their small characteristic length scale. In these cases, the flow of an incompressible Newtonian viscous fluid can be modelled by the Stokes system of equations, which states a balance between the pressure and the viscous-shear forces. In terms of a characteristic velocity $U$, a length scale $h$ and pressure $\mu U / h$, the Stokes system of equation can be written in dimensionless form as:

where

$$
\frac{\partial u_{i}}{\partial x_{i}}=0 \quad \frac{\partial \sigma_{i j}}{\partial x_{j}}=0
$$

$$
\sigma_{i j}=-p \delta_{i j}+\left(\frac{\partial u_{i}}{\partial x_{j}}+\frac{\partial u_{j}}{\partial x_{i}}\right)
$$

$\vec{u}$ being the velocity vector, $p$ the pressure and $\delta_{i j}$ the Kronecker delta.

Under these confined flow configurations, appropriate velocity slip conditions at the wall surfaces must be prescribed in order to employ continuum models for the description of the flow field. The Navier's slip boundary condition states that the relative tangential fluid velocity, $u_{t}^{f}$, with respect to the tangential wall velocity, $U_{t}^{w}$, is directly proportional to the tangential projection of the local shear rate, $\dot{\gamma}_{t}$, as given by equation (3). The proportional constant is called slip length, $L_{s}$ (dimensionless slip length in our case), and represents the hypothetical outward distance at the wall needed to satisfy the no-slip flow condition [12].

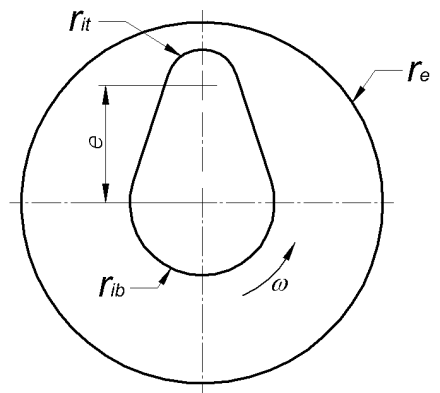

Figure 1: $\quad$ Single rotor mixer geometry. 
Besides, when dealing with rarefied gases the effect of thermal creep or transpiration needs to be considered, as it relates the tangential fluid velocity with the tangential heat flux at the walls. In this way, the complete slip condition is described by:

$$
u_{t}^{f}-U_{t}^{w}=L_{s} \dot{\gamma}_{t}+L_{s T}\left(-q_{s}\right)
$$

with

$$
\dot{\gamma}_{t}=\left(\frac{\partial u_{i}}{\partial x_{j}}+\frac{\partial u_{j}}{\partial x_{i}}\right) n_{j} s_{i}=\left(\frac{\partial u_{i}}{\partial n} s_{i}+\frac{\partial u_{j}}{\partial s} n_{j}\right)
$$

where $n_{i}$ and $s_{i}$ are respectively the $i$ components of the normal and tangential vectors to a boundary surface and $q_{s}=-k \partial T / \partial s$ is the tangential heat flux at the boundary contours with $k$ as the thermal conductivity. In the above expression, $L_{S T}$ is a coefficient constant proportional to the product of the Reynolds number times the square of the Knudsen number and inversely proportional to the Eckert number.

For the implementation of this type of slip condition in the velocity integral representational formula for Stokes flows, the tangential shear rate at solid-fluid interfaces can be evaluated in terms of the surface traction force $\bar{f}$, which tangential projection can be expressed as:

$$
\begin{gathered}
f_{t}=f_{i} s_{i}=\sigma_{i j} n_{j} s_{i}=\left[-p \delta_{i j} n_{i} s_{i}+\left(\frac{\partial u_{i}}{\partial x_{j}}+\frac{\partial u_{j}}{\partial x_{i}}\right) n_{j} s_{i}\right] \\
=\left(\frac{\partial u_{i}}{\partial x_{j}}+\frac{\partial u_{j}}{\partial x_{i}}\right) n_{j} s_{i}=\dot{\gamma}_{t}
\end{gathered}
$$

where for consistence with our formulation, the above expression has been written in dimensionless form, with a characteristic traction force $\mu U / h$.

To complete the boundary conditions at the solid surfaces, the following nonflux condition across the boundaries needs to be considered:

$$
u_{i}(x) n_{i}(x)=U_{n}^{w}(x) \text {, at any points } \mathrm{x} \text { on the boundary surfaces }
$$

where the following integral relation needs to be satisfied, according to the conservation of mass for an incompressible fluid:

$$
\int_{S=S_{i n t} \cup S_{\text {ext }}} U_{n}^{w}(x) d S_{x}=0
$$

with $S$ as the union of the external and internal surfaces, $S_{\text {ext }}$ and $S_{\text {int }}$, respectively. In our case for micro mixers with external stationary housing, $U_{n}^{w}(x)=U_{t}^{w}(x)=0$ at $x \in S_{\text {ext }}$. 


\section{Boundary integral formulation for slip Stokes flows}

The Stokes velocity field has the following direct integral representation formulae for an arbitrary point $x$ in a closed domain $\Omega$ filled with a Newtonian fluid [13]:

$$
c(x) u_{i}(x)-\int_{S} K_{i j}(x, y) u_{j}(y) d S_{y}+\int_{S} u_{i}^{j}(x, y) f_{j}(y) d S_{y}=0
$$

where $c$ is a constant dependent on the position of the source point. For internal points $c=1$, for point at a smooth boundaries $c=1 / 2$ and for external points $c=0$.

The kernels of the above integral representational formula are the Stokeslet and its corresponding surface tractions or Stresslet, which two dimension expressions are given by:

$$
\begin{aligned}
& u_{i}^{j}(x, y)=-\frac{1}{4 \pi}\left[\ln \left(\frac{1}{r}\right) \delta_{i j}+\frac{\left(x_{i}-y_{i}\right)\left(x_{j}-y_{j}\right)}{r^{2}}\right] \\
& K_{i j}(x, y)=-\frac{1}{\pi} \frac{\left(x_{i}-y_{i}\right)\left(x_{j}-y_{j}\right)\left(x_{k}-y_{k}\right) n_{k}(y)}{r^{4}}
\end{aligned}
$$

$r$ being the Euclidean distance between point $x$ and $y$, i.e. $r=|x-y|$.

Substituting the above slip condition with consideration of thermal jump, equation (3), into the normal and tangential projections of the Stokes velocity field, the following system of integral equations for the normal and tangential components of the unknown surface traction is found:

$$
\begin{aligned}
\int_{S} u_{i}^{j}(x, y)\left(f_{n}(y)\right. & \left.n_{j}(y)+f_{t}(y) s_{j}(y)\right) n_{i}(x) d S_{y} \\
& -\int_{S} K_{i j}(x, y) L_{S} f_{t}(y) s_{j}(y) n_{i}(x) d S_{y}=-c(x) U_{n}^{w} \\
& +\int_{S} K_{i j}(x, y)\left(U_{j}^{w}(y)\right) n_{i}(x) d S_{y} \\
& +\int_{S} K_{i j}(x, y)\left(L_{s T} \frac{\partial T(y)}{\partial s} s_{j}(y)\right) n_{i}(x) d S_{y}
\end{aligned}
$$

and 


$$
\begin{aligned}
c(x) L_{S} f_{t}(x)+\int_{S} & u_{i}^{j}(x, y)\left(f_{n}(y) n_{j}(y)+f_{t}(y) s_{j}(y)\right) s_{i}(x) d S_{y} \\
& -\int_{S} K_{i j}(x, y) L_{S} f_{t}(y) s_{j}(y) s_{i}(x) d S_{y} \\
& =-c(x) U_{t}^{w} \\
& +\int_{S} K_{i j}(x, y)\left(U_{j}^{w}(y)\right) s_{i}(x) d S_{y} \\
& +\int_{S} K_{i j}(x, y)\left(L_{s T} \frac{\partial T(y)}{\partial s} s_{j}(y)\right) s_{i}(x) d S_{y}
\end{aligned}
$$

The limiting value of the integral kernels in (10) and (11) as the radius $r$ tends to zero is given by:

$$
\begin{aligned}
& K_{i j}(x, y) n_{j}(y) n_{i}(x)=-\frac{r^{2}}{\pi}\left(\frac{1}{r} \frac{\partial r}{\partial n_{y}}\right)^{2}\left(\frac{1}{r} \frac{\partial r}{\partial n_{x}}\right)=-\frac{r^{2}}{\pi}\left(\frac{k(x)}{2}\right)^{3} \rightarrow 0 \\
& K_{i j}(x, y) s_{j}(y) n_{i}(x)=K_{i j}(x, y) n_{j}(y) s_{i}(x)=-\frac{r}{\pi} \frac{\partial r}{\partial s_{x}}\left(\frac{1}{r} \frac{\partial r}{\partial n_{y}}\right)^{2} \\
& =-\frac{r}{\pi}(\sin (\vec{n}, \vec{r}))\left(\frac{k(x)}{2}\right)^{2} \rightarrow 0 \\
& u_{i}^{j}(x, y) n_{j}(y) s_{i}(x)=u_{i}^{j}(x, y) s_{j}(y) n_{i}(x)=-\frac{r}{4 \pi} \frac{\partial r}{\partial s_{y}}\left(\frac{1}{r} \frac{\partial r}{\partial n_{x}}\right) \\
& =-\frac{r}{4 \pi}(\sin (\vec{n}, \vec{r}))\left(\frac{k(x)}{2}\right) \rightarrow 0 \\
& u_{i}^{j}(x, y) n_{j}(y) n_{i}(x)=-\frac{1}{4 \pi}\left[\ln \left(\frac{1}{r}\right)+r^{2}\left(\frac{1}{r} \frac{\partial r}{\partial n_{y}}\right)\left(\frac{1}{r} \frac{\partial r}{\partial n_{x}}\right)\right] \\
& =-\frac{1}{4 \pi}\left[\ln \left(\frac{1}{r}\right)-r^{2}\left(\frac{k(x)}{2}\right)^{2}\right] \rightarrow-\frac{1}{4 \pi} \ln \left(\frac{1}{r}\right) \\
& u_{i}^{j}(x, y) s_{j}(y) s_{i}(x)=-\frac{1}{4 \pi}\left[\ln \left(\frac{1}{r}\right)+\frac{\partial r}{\partial s_{y}} \frac{\partial r}{\partial s_{x}}\right]=-\frac{1}{4 \pi}\left[\ln \left(\frac{1}{r}\right)-\cos ^{2}(\vec{s}, \vec{r})\right] \\
& =-\frac{1}{4 \pi}\left[\ln \left(\frac{1}{r}\right)-\left(1-\cos ^{2}(\vec{n}, \vec{r})\right)\right] \\
& =-\frac{1}{4 \pi}\left[\ln \left(\frac{1}{r}\right)-1+r^{2}\left(\frac{k(x)}{2}\right)^{2}\right] \rightarrow-\frac{1}{4 \pi}\left(\ln \left(\frac{1}{r}\right)-1\right)
\end{aligned}
$$

with only logarithmic singularities on $u_{i}^{j}(x, y) n_{j}(y) n_{i}(x)$ and $u_{i}^{j}(x, y) s_{j}(y) s_{i}(x)$.

In the above limiting values, the term $\partial r / r \partial n$ tends to $k(x) / 2$ as the radius $r$ tends to zero, where $k(x)$ is the curvature at a point $x$ on the boundaries (see Courant and Hilbert [14, Vol. 2, page 299]). 
To solve equations (10) and (11), it is necessary to know the temperature field with the aim of obtaining the temperature gradient in the tangential direction at boundary contours. The temperature field is found by solving the energy equation, written here in dimensionless form as:

$$
P e\left(\frac{\partial T}{\partial t}+u_{j} \frac{\partial T}{\partial x_{j}}\right)=\frac{\partial^{2} T}{\partial x_{j}^{2}}
$$

where $P e=\left(U^{3} \mu / \rho g k\right)^{1 / 2}$ is the Peclet number, which can be expressed in terms of the Reynolds number through the Prandtl number as $\operatorname{Pr}=P e / R e=\mu / \rho k$. For cases of $\operatorname{Pr}=O(1)$, our previous assumption of small Reynolds number implies that $P e \ll 1$, reducing the above energy equation (at the first order of approximation in $P e$ ) to the Laplace equation, i.e. quasi-static approximation, the integral representation formula of which is:

$$
c(x) T(x)=\int_{S} \frac{\partial \phi(x, y)}{\partial n} T(y) d S_{y}-\int_{S} \phi(x, y) \frac{\partial T(y)}{\partial n} d S_{y}
$$

The kernels of the above integral representational formula are $\phi(r)=$ $-\ln (r) / 2 \pi$ for the surface single layer potential and $\partial \phi(x, y) / \partial n=$ $\left(x_{i}-y_{i}\right) n_{i}(y) / 2 \pi r^{2}=\cos (\vec{n}, \vec{r}) / 2 \pi r$ for the surface double layer potential, which are weak singular and regular, respectively (see our previous limiting values as $r$ tends to zero).

In the slip flow region, the temperature field is also subject to a jump condition at the boundary contours, [14], given by the radiation condition

$$
T^{f}-T^{w}=L_{T}\left(-q_{n}\right)
$$

with $q_{n}=-k \partial T / \partial n$ as the normal heat flux to the surface and $L_{T}$ as a coefficient constant function of the Knudsen and Prandtl numbers and the energy accommodation coefficient. The above jump condition represents a Robin type boundary condition for the temperature field. Substituting equation (14) into the integral representational formula for the temperature, equation (13), leads to the following boundary integral equation for the unknown normal flux:

$$
\begin{gathered}
c(x) L_{T} \frac{\partial T(x)}{\partial n}-\int_{S} \frac{\partial \phi(x, y)}{\partial n} L_{T} \frac{\partial T(y)}{\partial n} d S_{y}+\int_{S} \phi(x, y) \frac{\partial T(y)}{\partial n} d S_{y} \\
=-c(x) T^{w}(x)+\int_{S} \frac{\partial \phi(x, y)}{\partial n} T^{w}(y) d S_{y}
\end{gathered}
$$

The above integral equation can be solved by a classical BEM procedure; the quadratic scheme is the present work. The obtained surface heat flux can be substituted into the temperature jump condition (14) to find the corresponding fluid temperature at contact with the solid surfaces. 
The tangential heat flux at the boundary contours is obtained by taking the limiting value of the gradient of the temperature field, at an internal point, approaching a boundary point, and then multiplying the resulting expression by the surface tangential vector. In this way, the surface gradient of the temperature field is given by:

$$
c(x) \frac{\partial T(x)}{\partial x_{j}}=\int_{S} \frac{\partial \phi(x, y)}{\partial x_{j}} \frac{\partial T(y)}{\partial n} d S_{y}-\int_{S} \frac{\partial^{2} \phi(x, y)}{\partial x_{j} \partial n} T(y) d S_{y}
$$

where the first integral is a singular integral of the Cauchy type and the second a hyper singular integral. However, by multiplying the above relation by the surface tangential vector, the resulting integral relation for the tangential derivative only possesses singularities of the Cauchy type:

$$
\begin{aligned}
& c(x) \frac{\partial T(x)}{\partial s} \\
& =\int_{S} \frac{\partial \phi(x, y)}{\partial s} \frac{\partial T(y)}{\partial n} d S_{y} \\
& -\int_{S} \frac{\partial^{2} \phi(x, y)}{\partial s \partial n} T(y) d S_{y}
\end{aligned}
$$

where the limiting value of the above kernels as the distance $r$ tends to zero is given by:

$$
\begin{gathered}
\frac{\partial \phi(x, y)}{\partial s}=-\frac{1}{2 \pi} \frac{\partial \ln (r)}{\partial x_{j}} s_{j}=-\frac{\left(x_{j}-y_{j}\right)}{2 \pi r^{2}} s_{j}=\frac{1}{2 \pi} \frac{\sin (\vec{n}, \vec{r})}{r} \rightarrow \frac{1}{r} \\
\frac{\partial^{2} \phi(x, y)}{\partial s \partial n}=\frac{\delta_{i j}}{2 \pi r^{2}} n_{j} s_{i}-\frac{\left(x_{j}-y_{j}\right)\left(x_{i}-y_{i}\right)}{\pi r^{4}} n_{j} s_{i} \\
=\frac{n_{j}(y) s_{i}(x)}{2 \pi r^{2}}-\frac{1}{\pi} \frac{\cos (\vec{n}, \vec{r})}{r} \frac{\sin (\vec{n}, \vec{r})}{r} \rightarrow-\frac{1}{\pi} \frac{k(x)}{2} \frac{\sin (\vec{n}, \vec{r})}{r}
\end{gathered}
$$

The evaluation of the above integrals needs to be considered in the sense of Cauchy principal value. Various regularization methods to reduce the order of singularity of this type of integrals are available in the literature; most of them are based on expanding the kernel around the singular point and subtraction of the most singular part as originally suggested by Mikhlin [16]. A simple alternative is to find the integral representational formulae of a known potential field, with the same singularity as the field in consideration. Subtraction of both integral equations removes the most singular part of the integral operators (for more details see [16]). In general, this is not a simple task, but in the present case it is possible to define the potential 


$$
\tilde{T}(x)=\frac{\partial T(p)}{\partial x_{j}}\left(x_{j}-x(p)_{j}\right)+T(p),
$$

solution of the Laplace equation $\nabla^{2} \widetilde{T}=0$, with values of the constants $T(p)$ and $\partial T(p) / \partial x_{j}$ equal to the values of the temperature field (13) and its gradient at a given evaluation point $\vec{x}(p)$. Therefore, at the evaluation point $\vec{x}(p), \tilde{T}(p)=$ $T(p)$ and $\frac{\partial \tilde{T}(p)}{\partial x_{j}}=\frac{\partial T(p)}{\partial x_{j}}$. By subtracting the integral representation formula of the tangential derivative of both fields, we obtain

$$
\begin{aligned}
c(x)\left(\frac{\partial T(x)}{\partial s}-\frac{\partial \tilde{T}(x)}{\partial s}\right) \\
\quad=\int_{S} \frac{\partial \phi(x, y)}{\partial s}\left(\frac{\partial T(y)}{\partial n}-\frac{\partial \tilde{T}(y)}{\partial n}\right) d S_{y} \\
-\int_{S} \frac{\partial^{2} \phi(x, y)}{\partial s \partial n}(T(y)-\tilde{T}(y)) d S_{y}
\end{aligned}
$$

At an evaluation point $\vec{x}(p)$, the above integral relation reduces to the following regular integral equation:

$$
\begin{array}{r}
\int_{S} \frac{\partial^{2} \phi(p, y)}{\partial s \partial n}\left(T(y)-T(p)-\frac{\partial T(p)}{\partial x_{j}}\left(y_{j}-p_{j}\right)\right) d S_{y} \\
=\int_{S} \frac{\partial \phi(p, y)}{\partial s}\left(\frac{\partial T(y)}{\partial n}-\frac{\partial T(p)}{\partial n}\right) d S_{y}
\end{array}
$$

Given the values of the temperature $T$ and its normal derivative $\frac{\partial T}{\partial n}$, obtained from (14) and (15), equation (19) provides a linear relationship between the directional derivatives of the temperature at the evaluation point $\vec{x}(p)$, i.e. between the values of $\frac{\partial T(p)}{\partial x_{1}}$ and $\frac{\partial T(p)}{\partial x_{2}}$. A second relation between these two values is given by the known normal derivative at $\vec{x}(p)$, i.e. $\frac{\partial T(p)}{\partial n}=\frac{\partial T(p)}{\partial x_{1}} n_{1}+$ $\frac{\partial T(p)}{\partial x_{2}} n_{2}$. From these two expressions, it is possible to obtain the directional derivatives of the temperature field at each boundary point and find the corresponding tangential derivative $\frac{\partial T(p)}{\partial S}=\frac{\partial T(p)}{\partial x_{1}} S_{1}+\frac{\partial T(p)}{\partial x_{2}} S_{2}$ to be used in the integral equations (10) and (11) for the normal and tangential components of the surface velocity in order to determine the corresponding effect of the thermal creep on the flow field.

Figure 2 shows the obtained fluid temperature distribution at the rotor and housing boundaries of the single rotor mixer given in Figure 1, for different constant temperature at the solid walls. Two different cases are reported in the figure, corresponding to a hotter and colder rotor, i.e. $T_{r i}>T_{r e}$ and $T_{r e}>T_{r i}$ 
respectively. In Figure 3, we report the corresponding velocity fields inside the mixer in the case of a stationary rotor, corresponding to only a thermal creep fluid motion. Two symmetric recirculation regions are formed around the rotor head, where the direction of the recirculation motion changes according to the direction of the heat flux, i.e. $T_{r i}>T_{r e}$ or $T_{r e}>T_{r i}$. Similar recirculation patterns are reported by Lockerby et al. [12] in the case of eccentric circular cylinders, however in their analysis they do not use a jump temperature condition, as in (13), but instead they use a high order thermal stress slip condition, where the tangential velocity is proportional to the tangential derivative of the normal heat flux.

In order to see the thermal creep effect on the recirculation pattern at the rotor head of a rotating mixer, in Figure 4 is reported the obtained flow fields corresponding to $L_{S T}=0.1,1$ and 10 . As can be observed from the figure, as the value of $L_{S T}$ increases the recirculation and the slip velocity at the rotor head are strongly affected by the thermal creep until the value of $L_{S T}$ becomes too large so that the thermal creep effect dominates the flow behaviour and a recirculation zone appears at the sides of the rotor head, as those observed in Figure 3 for the case of only thermal creep, but in the present case they are not located symmetrically. In addition, in the condition of very large value $L_{S T}$, the position of the recirculation zones changes with the direction of the heat flux.

\section{Conclusions}

In this work, the thermal creep effect at micro scale flows has been evaluated. This phenomenon usually takes place due to micro fluid flow of rarefied gases and could affect the velocity field due to a heat flux and a temperature difference in the domain. An integral equation approach based on the normal and tangential projections of the direct boundary integral representational formula for the Stokes velocity field is developed for the numerical simulation of creeping flow

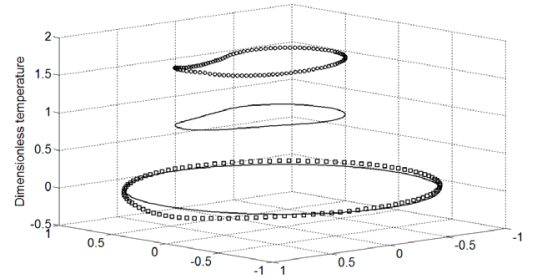

(A)

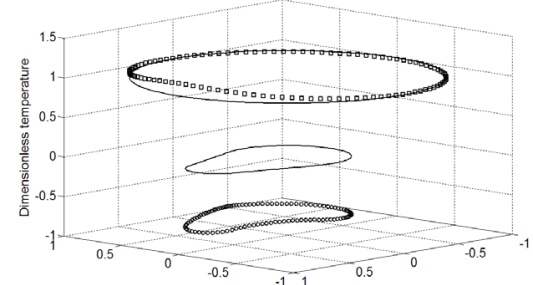

(B)

Figure 2: $\quad$ Temperature profiles at single rotor boundaries for $L_{T}=1: T_{r i}>$ $T_{r e}$ (A) and $T_{r e}>T_{r i}$, (B). Solid lines represent the wall temperatures. 


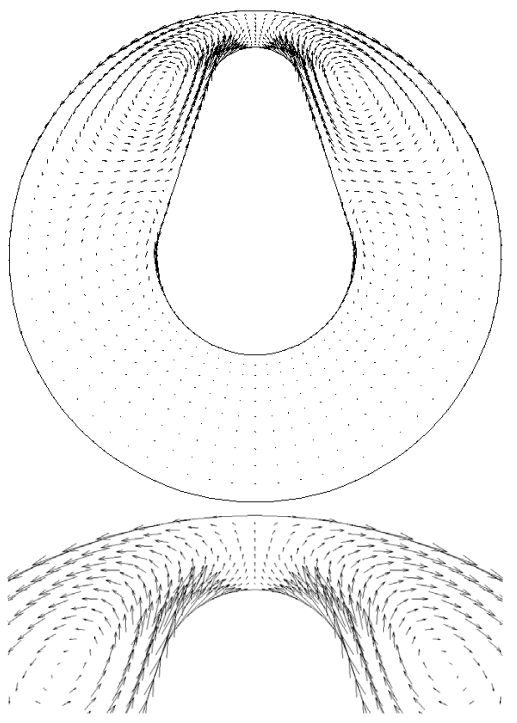

(A)

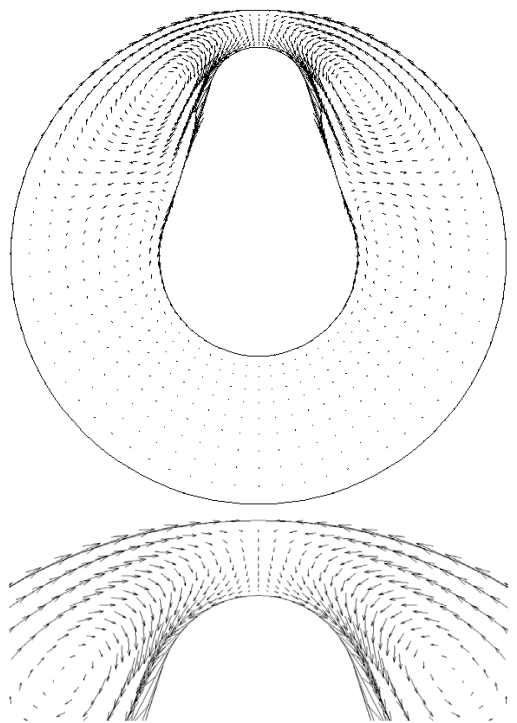

(B)

Figure 3: Vector velocities for $L_{s}=0.5, L_{T}=1$ and $L_{s T}=10$ and $T_{r i}>T_{r e}$ (A) and $T_{r e}>T_{r i}$, (B), without considering rotational velocity at the inlet surface.

under linear slip boundary conditions and applied to analyze the performance of a single rotor mixer subjected to thermal creep. The slip condition was included in the boundary integral formulation by expressing the tangential shear rate in terms of the tangential component of the surface traction vector. The projection of the velocity integral representational formula on the normal and tangential directions smoothes the singularity of the integral kernels, resulting only in a weak singular kernel of the logarithm type, which can be numerically integrated by using Telles' transformation and standard Gaussian Quadrature formulae.

The integral formulation has been modified to account for the thermal effect, which is a function of the tangential heat flux. For the latter, an integral representational formulae was obtained by taking the limiting value of the gradient of the temperature field and multiplying the resulting relation by the surface tangential vector, presenting only singularities of the Cauchy type. The order of singularity of this type of integral was reduced by finding the integral representational formulae of a known potential field, with the same singularity as the field in consideration, reducing the expression to a regular integral equation at the evaluation point. The implementation of this formulation allowed us to observe that the thermal creep effect can modify the recirculation pattern at the rotor head of a rotating mixer dominating the flow behaviour and leading to recirculation zones that appear at different sides of the rotor head depending on the direction of the heat flux (see Figure 4). 


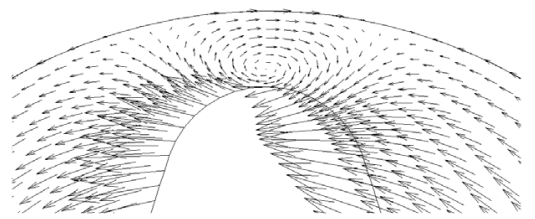

(A)

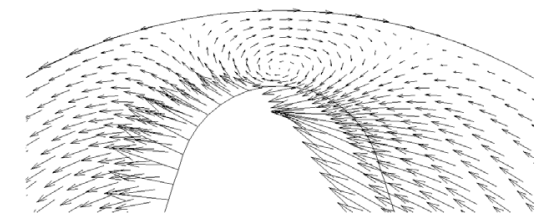

(B)

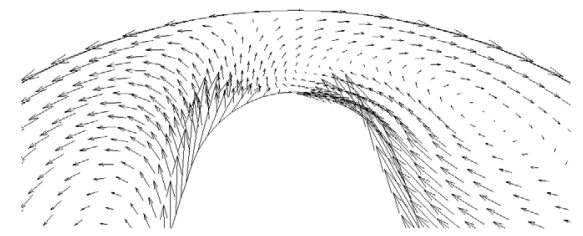

(C)

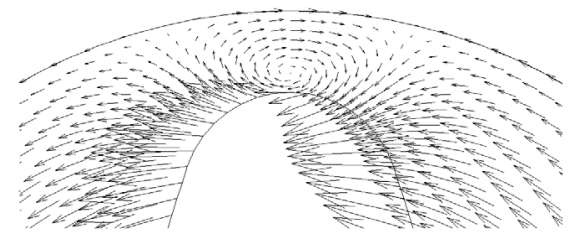

(D)

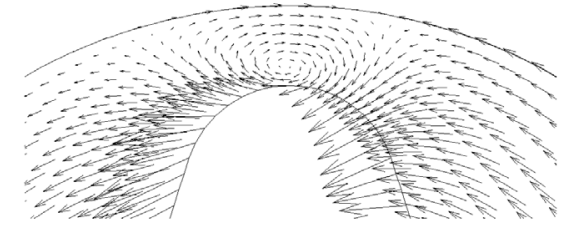

(E)

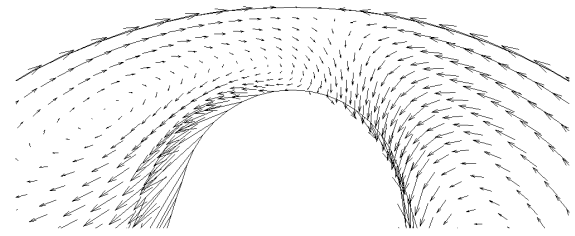

(F)

Figure 4: $\quad$ Vector velocities with $L_{S}=0.1$ and $L_{T}=1, L_{s T}=0.1(\mathrm{~A}), L_{s T}=1$ (B), $L_{s T}=10$ (C), $L_{s T}=0.1$ (D), $L_{s T}=1$ (E), $L_{s T}=10(\mathrm{~F})$. Results in (A)-(C) are for $T_{r i}>T_{r e}$, while in (D)-(F) are for $T_{r e}>T_{r i}$. 


\section{Acknowledgements}

The first author acknowledges the support of the $\mathrm{PhD}$ program at the Universidad Pontificia Bolivariana and COLCIENCIAS, Colombia, under the support provided with the project "Desarrollo de geometrías para aplicación industrial en microintercambiadores de calor" code 1210-479-21999, contract 436-2008.

\section{References}

[1] E.A. Mansur, Y. Mingxing, W. Yundong and D. Youyuan, A state-of-theart review of mixing in microfluidic mixers, Chin. J. Chem. Eng., 16 (2008), 503-516.

[2] V. Hessel, H. Lwe, F. Schnfeld, Micromixers: a review on passive and active mixing principles, Chemical Engineering Science, 60 (2005), 24792501.

[3] G. Hu and D. Li, Multiscale phenomena in microfluidics and nanofluidics, Chemical Engineering Science, 62 (2007), 3443-3454.

[4] Jae-Yong Park, Yong-Dae Kim, Sang-Rak Kim, Seog-Young Han and JooSungMaeng, Robust design of an active micro-mixer based on the Taguchi method, Sensors and Actuators B, 129 (2008), 790-798.

[5] K.F. Lei and W.J. Li, A novel in-plane microfluidic mixer using vortex pumps for fluidic discretization, JALA, 13 (2008), 227-236.

[6] A. Goullet, I. Glasgow, and N. Aubry, Effects of microchannel geometry on pulsed flow mixing, Mech. Res. Commun., 33 (2006), 739-746.

[7] A.A. Mouza, C.-M. Patsa, F. Schnfeld, Mixing performance of a chaotic micro-mixer, Chemical Engineering Research and Design, 86 (2008), $1128-1134$.

[8] P. A. Thompson and S. M. Troian, A general boundary condition for liquid flow at solid surfaces, Nature, 389 (1997), 360-362.

[9] O.I. Vinogradova, Slippage of water over hydrophobic surfaces, Int. Journal of Miner. Process., 56 (1999), 31-60.

[10] C. Neto, D.R. Evans, E. Bonaccurso, H. Butt and V. Craig, Boundary slip in Newtonian liquids: a review of experimental studies, Rep. Progr. Phys., 68 (2005), 2859-2897.

[11] G. Karniadakis, A. Beskok and N. Aluru, "Microflows and Nanoflows: Fundamentals and Simulation," 1st edition, Springer, New York, 2005.

[12] D. Lockerby, J. M. Reese, D. R. Emerson and R. W. Barber. Velocity boundary condition at solid walls in rarefied gas calculations, Physical Review E 70, (2004), 017303-1 - 017303-9.

[13] H. Power and L.C. Wrobel, "Boundary Integral Methods in Fluid Mechanics," 1st edition, Computational Mechanics Publications, Southampton, 1995.

[14] R. Courant and D. Hilbert, Methods of mathematical physics, $3^{\text {rd }}$ edition, Cambridge University Press, Cambridge, 1962. 
28 Boundary Elements and Other Mesh Reduction Methods XXXII

[15] O. Aydin, M, Avc1. Heat and fluid flow characteristics of gases in micropipes. International Journal of Heat and Mass Transfer, 49, (2006), 1723-1730.

[16] S.G. Mikhlin, Multidimensional Singular Integrals and Integral Equations. Pergamon Press, New York, 1957.

[17] H. Chen, J. Jin, P. Zhang and P. Lu, Multi-variable non-singular BEM for 2-D potential problems, Tsinghua Science and Technology, Vol 10, 1, 4350. 